\title{
On the Multiple-Access Capacity of Multitone-CDMA Communications
}

\author{
Kun-Wah Yip, Member, IEEE, Xi Zhang, Tung-Sang Ng, Senior Member, IEEE, and \\ Jiangzhou Wang, Senior Member, IEEE
}

\begin{abstract}
This letter derives a bit-error-probability expression for an asynchronous multitone-code-division multiple access (CDMA) system and shows that the multiple-access (MA) capacity of this system is identical to that of a conventional (single-carrier) direct-sequence spread-spectrum system under conditions of equal bandwidth and equal bit rate. It is also shown that using more subcarriers to increase the processing gain does not increase the MA capacity.
\end{abstract}

Index Terms-Multiple-access capacity, multitone-CDMA, OFDM.

\section{INTRODUCTION}

$\mathbf{M}$ ULTITONE code-division multiple access (MTCDMA) technique [1] combines both orthogonal frequency division multiplexing (OFDM) and direct-sequence spread-spectrum (DSSS) technique in such a way that, by increasing the number of subcarriers used, the processing gain of a MT-CDMA system can be made substantially greater than that of a conventional (single-carrier) DSSS system under the same-bandwidth condition. It has been expected that this greater processing gain could lead to a greater reduction in the multiple-access interference (MAI) so that a MT-CDMA system could support a larger number of users than a conventional DSSS system [2]. However, based on the bit error probability derived in Section II, we show in Section III that, under equal-bandwidth and equal-bit-rate conditions, the multiple-access (MA) capacity of an asynchronous MT-CDMA system is identical to that of a conventional DSSS system and does not increase by an increase of the number of subcarriers used. Despite the MA capacity is not increased, the greater processing gain of a MT-CDMA signal does have other advantages such as more effective suppression of the narrowband interference. The result of this letter thus indicates that, for an effective utilization of the greater processing gain, system designers should focus on these advantages rather than on increasing the MA capacity.

Manuscript received January 11, 1999. The associate editor coordinating the review of this letter and approving it for publication was Prof. M. K. Tsatsanis. This work was supported by the Hong Kong Research Grants Council and by the University Research Committee of the University of Hong Kong, Hong Kong.

The authors are with the Department of Electrical and Electronic Engineering, University of Hong Kong, Pokfulam Road, Hong Kong (e-mail: kwyip@eee.hku.hk; xzhang@eee.hku.hk; tsng@eee.hku.hk; jwang@eee. hku.hk).

Publisher Item Identifier S 1089-7798(00)01245-X.

\section{System Model AND ERROR PERFORMANCE}

Consider a MT-CDMA system with $K$ users transmitting their signals at equal power and equal bit rate. At the transmitter, the incoming bit stream having a rate of $M / T_{b}$ bits per second is first serial-to-parallel converted into $M$ lower-rate bit substreams each of which has a bit duration of $T_{b}$ seconds. Assume that rectangular symbol waveform and binary phase shift keying are used. The $M$ substreams are, respectively, modulated onto $M$ orthogonal subcarriers that are equally spaced by $f_{\Delta} \mathrm{Hz}$. Orthogonality among subcarriers is maintained by setting $f_{\Delta}=$ $1 / T_{b}$. The data-modulated subcarriers are multiplexed and the resultant multiplexed signal is spread by a spreading sequence of length $N$. The chip duration, $T_{c}$, is given by $T_{c}=T_{b} / N$. The spread signal is then transmitted over the channel. Note that in the special case of $M=1$, the MT-CDMA system is essentially a conventional DSSS system.

The received signal is the sum of $K$ transmitted signals corrupted by additive white Gaussian noise (AWGN) having a twosided power spectral density $N_{0} / 2$. It is assumed that the receiver is intended to detect the $L$ th-user signal. The complex envelope of the received signal is given by

$$
r(t)=n(t)+\sum_{k=1}^{K} \sqrt{2 P_{b}} e^{j \theta_{k}} \sum_{\substack{i=-\infty \\ \cdot u_{k}\left(t-i T_{b}-\tau_{k}\right)}}^{\infty-1} \sum_{i=0}^{(k)} e^{j 2 \pi m f_{\Delta}\left(t-\tau_{k}\right)}
$$

where $n(t)$ is the baseband-equivalent AWGN; $P_{b}$ is the signal power per bit (so that $M P_{b}$ is the transmitted signal power); $\theta_{k}$ is the random phase introduced by the $k$ th-user transmitter; $b_{i, m}^{(k)} \in$ $\{+1,-1\}$ is the $i$ th bit transmitted on the $m$ th subcarrier of the $k$ th-user signal; $\tau_{k}$ is the delay of the $k$ th-user signal relative to the $L$ th-user signal; and

$$
u_{k}(t)=\sum_{n=0}^{N-1} a_{n}^{(k)} \psi\left(t-n T_{c}\right)
$$

is the $k$ th-user spectral spreading waveform. In (2), $\left(a_{0}^{(k)}\right.$, $\left.a_{1}^{(k)}, \cdots, a_{N-1}^{(k)}\right)$ is the $k$ th user spreading sequence satisfying $\left|a_{n}^{(k)}\right|=1$, and $\psi(t)$ is the chip waveform given by $\psi(t)=1$ for $t \in\left[0, T_{c}\right)$ and $\psi(t)=0$ otherwise. Asynchronous signal transmission is considered so that $\tau_{L}$ is given by $\tau_{L}=0$ and $\tau_{k}$ 's, $k \neq L$, are modeled as mutually independent random variables uniformly distributed over $\left[0, T_{b}\right)$. The received signal is processed by a bank of $M$ matched filters each of which detects the bits transmitted on a particular subcarrier. 
Assume that coherent detection is used and $b_{i, \tilde{m}}^{(L)}$ is to be detected. The desired matched-filter output, $Z_{\tilde{i}, \tilde{m}}$, is given by

$Z_{\tilde{i}, \tilde{m}}=\frac{1}{\sqrt{2 P_{b}} T_{b}} \int_{\tilde{i} T_{b}}^{(\tilde{i}+1) T_{b}} r(t) u_{L}^{*}\left(t-\tilde{i} T_{b}\right) e^{-j 2 \pi \tilde{m} f_{\Delta} t} e^{-j \theta_{L}} d t$.

The decision variable for $b_{\tilde{c}, \tilde{m}}^{(L)}$ is the sign of the real part of $Z_{\tilde{i}, \tilde{m}}$. Although we use $M$ matched filters to process $r(t)$ for theoretical modeling, in practical implementations efficient DFT algorithms are often employed [3]. Let $E_{b}=P_{b} T_{b}$ denote the bit energy. Substituting (1) into (3) gives

$$
Z_{\tilde{i}, \tilde{m}}=\eta+b_{\tilde{i}, \tilde{m}}^{(L)}+\sum_{k=1, k \neq L}^{K} J_{k}
$$

where $\eta$ is the zero-mean complex Gaussian noise with a variance $E\left\{\eta \eta^{*}\right\}=\left(E_{b} / N_{0}\right)^{-1}$, and

$$
\begin{aligned}
J_{k}= & \frac{1}{N} e^{j\left(\theta_{k}-\theta_{L}\right)} \sum_{m=0}^{M-1} e^{-j 2 \pi m f_{\Delta} \tau_{k}} e^{j 2 \pi(m-\tilde{m}) f_{\Delta} \tilde{i} T_{b}} \\
& \cdot\left[b_{\tilde{i}-1, m}^{(k)} S_{k, L}\left((m-\tilde{m}) f_{\Delta}\right)+b_{\tilde{i}, m}^{(k)} \hat{S}_{k, L}\left((m-\tilde{m}) f_{\Delta}\right)\right]
\end{aligned}
$$

is the MAI due to the $k$ th user. In (5),

$$
S_{k, L}(\mu)=T_{c}^{-1} \int_{0}^{\tau_{k}} e^{j 2 \pi \mu t} u_{k}\left(t+T_{b}-\tau_{k}\right) u_{L}^{*}(t) d t
$$

and

$$
\hat{S}_{k, L}(\mu)=T_{c}^{-1} \int_{\tau_{k}}^{T_{b}} e^{j 2 \pi \mu t} u_{k}\left(t-\tau_{k}\right) u_{L}^{*}(t) d t
$$

Let

$$
\begin{aligned}
& C_{k, L}(q ; \xi) \\
& = \begin{cases}\sum_{i=0}^{N-1-q} a_{i}^{(k)} a_{i+q}^{(L) *} e^{j 2 \pi(i+q) \xi}, & 0 \leq q \leq N-1 \\
\sum_{i=-q}^{N-1} a_{i}^{(k)} a_{i+q}^{(L) *} e^{j 2 \pi(i+q) \xi}, & -(N-1) \leq q<0 \\
0, & |q| \geq N .\end{cases}
\end{aligned}
$$

Let $w_{k} \in\{0,1, \cdots, N-1\}$ and $\hat{\tau}_{k} \in\left[0, T_{c}\right)$ be the quotient and remainder, respectively, when $\tau_{k}$ is divided by $T_{c}$. Note that $\tau_{k}=w_{k} T_{c}+\hat{\tau}_{k}$. Substituting (2) into (6) and (7) followed by some algebraic manipulations, we get

$$
\begin{aligned}
& S_{k, L}(\mu)=R\left(\hat{\tau}_{k} ; \mu\right) \cdot C_{k, L}\left(-\left(N-w_{k}-1\right) ; \mu T_{c}\right) \\
& +\hat{R}\left(\hat{\tau}_{k} ; \mu\right) \cdot C_{k, L}\left(-\left(N-w_{k}\right) ; \mu T_{c}\right) \\
& \hat{S}_{k, L}(\mu)=R\left(\hat{\tau}_{k} ; \mu\right) \cdot C_{k, L}\left(w_{k}+1 ; \mu T_{c}\right) \\
& +\hat{R}\left(\hat{\tau}_{k} ; \mu\right) \cdot C_{k, L}\left(w_{k} ; \mu T_{c}\right)
\end{aligned}
$$

We apply the Gaussian approximation on the MAI in the computation of the bit error probability, $P_{e}$. This approximation is justified for a moderate value of $K$. It follows from (4) that

$$
P_{e}=Q\left(\left\{\left(\frac{2 E_{b}}{N_{0}}\right)^{-1}+\frac{1}{2} \sum_{k=1, k \neq L}^{K} \operatorname{var}\left\{J_{k}\right\}\right\}^{-1 / 2}\right)
$$

where $Q(x)=(2 \pi)^{-1 / 2} \int_{x}^{\infty} e^{-t^{2} / 2} d t$ is the standard $Q$ function, and

$$
\begin{aligned}
\operatorname{var}\left\{J_{k}\right\}=\frac{1}{N^{2}} \sum_{m=0}^{M-1}[ & V_{1}\left(V_{4}+V_{6}\right)+V_{2}\left(V_{5}+V_{7}\right) \\
& \left.+2 \operatorname{Re}\left(V_{3} V_{8}+V_{3} V_{9}\right)\right] .
\end{aligned}
$$

In (14)

$$
\begin{aligned}
& V_{1}=E\left\{\left|R\left(\hat{\tau}_{k} ; \mu\right)\right|^{2}\right\} \\
& V_{2}=E\left\{\left|\hat{R}\left(\hat{\tau}_{k} ; \mu\right)\right|^{2}\right\} \\
& V_{3}=E\left\{R\left(\hat{\tau}_{k} ; \mu\right) \cdot \hat{R}^{*}\left(\hat{\tau}_{k} ; \mu\right)\right\}, \\
& V_{4}=E\left\{\left|C_{k, L}\left(-\left(N-w_{k}-1\right) ; \mu T_{c}\right)\right|^{2}\right\} \\
& V_{5}=E\left\{\left|C_{k, L}\left(-\left(N-w_{k}\right) ; \mu T_{c}\right)\right|^{2}\right\} \\
& V_{6}=E\left\{\left|C_{k, L}\left(w_{k}+1 ; \mu T_{c}\right)\right|^{2}\right\} \\
& V_{7}=E\left\{\left|C_{k, L}\left(w_{k} ; \mu T_{c}\right)\right|^{2}\right\} \\
& V_{8}=E\left\{C_{k, L}\left(-\left(N-w_{k}-1\right) ; \mu T_{c}\right) C_{k, L}^{*}\left(-\left(N-w_{k}\right) ; \mu T_{c}\right)\right\} \\
& V_{9}=E\left\{C_{k, L}\left(w_{k}+1 ; \mu T_{c}\right) \cdot C_{k, L}^{*}\left(w_{k} ; \mu T_{c}\right)\right\}
\end{aligned}
$$

where $\mu=(m-\tilde{m}) f_{\Delta}$ and $E\{\cdot\}$ is the expectation taken over random variables $\hat{\tau}_{k}$ and $w_{k}$. Notice that $P_{e}$ is dependent on the choice of $\tilde{m}$.

\section{ANALYSIS OF THE MA CAPACITY}

The MA capacity may be defined as the number of users that can be supported at a given error performance. We are interested in comparing the MA capacity of a MT-CDMA system and that of a conventional DSSS system under conditions of equal bit transmission rate and equal bandwidth. This comparison is made easy since a MT-CDMA system reduces to a conventional DSSS system in the special case of $M=1$. We are also interested in comparing MA capacities for MT-CDMA systems using different values of $M$. We want to find out whether the MA capacity could be increased by an increase in the processing gain through the use of more subcarriers.

We assume that the MT-CDMA system and the corresponding conventional DSSS system have the same chip duration $T_{c}$. As a result, the bandwidth of a MT-CDMA signal is slightly greater than the signal bandwidth required for a conventional DSSS system. The difference in their bandwidths is small (see [1]) so that the equal-bandwidth condition can be considered satisfied. As $M$ subcarriers are used in the MT-CDMA system, under equal-bit-rate condition the bit duration $T_{b}$ of the MT-CDMA system is required to be $M$ times of that of the conventional DSSS system. Hence, the spreading-code length $N$ of the MT-CDMA system should also be $M$ times of that of the corresponding conventional DSSS system. The increase in the spreading-code length constitutes 
TABLE I

MAXIMUM AND Minimum VALUES OF $I$ FOR VARIOUS COMBINATIONS OF $M$ AND $N$ UndER THE CONDITION OF SAME $N / M$

\begin{tabular}{ccc}
\hline & \multicolumn{2}{c}{$I$} \\
$N / M$ & min. & $\max$. \\
\hline $16 / 1$ & 0.6667 & 0.6667 \\
$32 / 2$ & 0.6660 & 0.6660 \\
$64 / 4$ & 0.6655 & 0.6662 \\
$128 / 8$ & 0.6653 & 0.6662 \\
$256 / 16$ & 0.6651 & 0.6662 \\
$512 / 32$ & 0.6650 & 0.6662 \\
$1024 / 64$ & 0.6650 & 0.6662 \\
\hline $128 / 1$ & 0.6667 & 0.6667 \\
$256 / 2$ & 0.6667 & 0.6667 \\
$512 / 4$ & 0.6667 & 0.6667 \\
$1024 / 8$ & 0.6667 & 0.6667 \\
\hline
\end{tabular}

an increase in the processing gain of a MT-CDMA system. Define the basic processing gain $N_{b a}$ by

$$
N_{b a}=\frac{N}{M}
$$

which is a constant invariant to the choice of $M$. Note that $N_{b a}$ is also the spreading-code length of the conventional DSSS system. Since $M$ bits are transmitted concurrently, the power per bit $P_{b}$ for a MT-CDMA system is required to be $1 / M$ times of that of the conventional DSSS system. The net result of an $M$-fold increase in the bit duration and an $M$-fold reduction in the power per bit is that the bit energy values are the same for both MT-CDMA and conventional DSSS systems. Thus, we can compare the two systems at the same $E_{b} / N_{0}$ value.

Some insight can be obtained by considering random binary sequences as spreading sequences. It can be shown that, for random binary sequences and $k \neq L$,

$$
\begin{gathered}
E\left\{\left|C_{k, L}\left(q ; \mu T_{c}\right)\right|^{2}\right\}= \begin{cases}N-|q| & |q|<N \\
0 & |q| \geq N\end{cases} \\
E\left\{C_{k, L}\left(q_{1} ; \mu T_{c}\right) \cdot C_{k, L}^{*}\left(q_{2} ; \mu T_{c}\right)\right\}=0 \quad \text { for } q_{1} \neq q_{2} .
\end{gathered}
$$

It follows that $\operatorname{var}\left\{J_{k}\right\}=I / N_{b a}$ where

$$
\begin{aligned}
I=\frac{1}{M} \sum_{m=0}^{M-1}[ & E\left\{\left|R\left(\hat{\tau}_{k} ;(m-\tilde{m}) f_{\Delta}\right)\right|^{2}\right\} \\
& \left.+E\left\{\left|\hat{R}\left(\hat{\tau}_{k} ;(m-\tilde{m}) f_{\Delta}\right)\right|^{2}\right\}\right] .
\end{aligned}
$$

Table I lists the maximum and minimum values of $I$ obtained over $\tilde{m} \in\{0,1, \cdots, M-1\}$ for various combinations of $M$ and $N$ under the condition of same $N_{b a}$. It is apparent that $I \approx$ $2 / 3$ for all combinations. Therefore, $P_{e}$ in (13) can be closely approximated by

$$
P_{e} \approx Q\left(\left\{\left(\frac{2 E_{b}}{N_{0}}\right)^{-1}+\frac{K-1}{3 N_{b a}}\right\}^{-1 / 2}\right)
$$

Equation (19) is identical to the one obtained by Pursley [4] for computing the error probability of a conventional DSSS system. It follows that the MA capacity of a MT-CDMA system is the same as that of a conventional DSSS system when asynchronous signal transmission and random sequences are used. Also notice that $P_{e}$ for a MT-CDMA system depends only on the basic processing gain. Thus, using a larger number of subcarriers to increase the processing gain of the system does not reduce $P_{e}$ and hence does not increase the MA capacity.

\section{REFERENCES}

[1] L. Vandendorpe, "Multitone spread spectrum multiple access communications system in a multipath Rician fading channel," IEEE Trans. Veh. Technol., vol. 44, pp. 327-337, May 1995.

[2] S. Hara and R. Prasad, "Overview of multicarrier CDMA," IEEE Commun. Mag., vol. 35, no. 12, pp. 126-133, Dec. 1997.

[3] B. Hirosaki, "An orthogonally multiplexed QAM system using the discrete Fourier transform," IEEE Trans. Commun., vol. 29, pp. 982-989, July 1981.

[4] M. B. Pursley, "Performance evaluation for phase-coded spread-spectrum multiple-access communication-Part I: System analysis," IEEE Trans. Commun., vol. COM-25, pp. 795-799, Aug. 1977. 